Conclusions NSPA expression in the cell surface of kidney and liver cells and not the P0 provides a potential target for anti$\mathrm{P}$ pathogenic effects, which might contribute to lupus hepatitis and nephritis.

Funding Source(s): Programa de Financiamiento Basal (AFB 17/ 0005) and FONDECYT $\mathrm{N}^{\circ} 1160513$.

\section{TREATMENT OUTCOME IN LUPUS NEPHRITIS PATIENTS TREATED WITH MYCOPHENOLATE MOFETIL: FROM A REAL-WORLD CLINICAL PRACTICE}

Seung Min Jung*, Sung Soo Ahn, Sang-Won Lee, Jason Jungsik Song, Yong-Beom Park. Yonsei University College of Medicine

\subsection{6/lupus-2019-Ism.125}

Background Systemic lupus erythematosus (SLE) is autoimmune disorder often characterized by the development of glomerulonephritis. The use of mycophenolate mofetil (MMF) is highlighted as induction and maintenance therapy in lupus nephritis. We evaluated the treatment outcome of MMF in lupus nephritis patients from a real clinical practice.

Methods Patients with biopsy proven lupus nephritis (class III, IV, and V) between November 2005 and August 2017 in Severance Hospital were extracted, and those patients who were treated with MMF at least 3 months were included in this study. The remission rate of lupus nephritis and risk factors for failure of remission were evaluated using Kaplan-Meier analysis and Cox proportional hazards model.

Results Of 116 patients included in this study, 89 (76.7\%) patients achieved remission of lupus nephritis after treatment with MMF. The median time to remission was 4.2 months (interquartile range 0.9 9.1). Normal complement level, negative result of anti-dsDNA antibody, and nephrotic range proteinuria were risk factors for remission failure in univariate analysis $(p=0.017,0.001$, and 0.007 , respectively). Nephrotic range proteinuria and negative result of anti-dsDNA antibody are independently associated with remission failure in multivariate analysis (OR 3.19, $p=0.004$ and $O R$ 1.62, $p=0.028$, respectively).

Conclusions Patients with lupus nephritis showed a favourable clinical outcome after MMF treatment. However, additional therapy would be required in patients with nephrotic-range proteinuria and without anti-dsDNA antibody.

Funding Source(s): None

\section{ANTI-RIBOSOMAL P AUTOANTIBODIES ARE NOT A MARKER FOR LUPUS NEPHRITIS}

${ }^{1}$ Milena Mimica, 'Loreto Massardo, ${ }^{1}$ Marcela Bravo-Zehnder, ${ }^{1}$ Patricia Gajardo, ${ }^{2}$ Paula Burgos, ${ }^{3}$ Alfonso González. ${ }^{1}$ Centro de Biología Celular y Biomedicina (CEBICEM). Faculty of Science and Medicine, Universidad San Sebastián. Santiago, Chile; ${ }^{2}$ Faculty of Medicine, Pontificia Universidad Católica de Chile. Santiago, Chile; ${ }^{3}$ Centro de Biología Celular y Biomedicina. Faculty of Science and Medicine, Universidad San Sebastián. Santiago, Chile. Centro de Envejecimiento y Regeneración (CARE), Facultad de Ciencias, Pontificia Universidad Católica de Chile. Santiago, Chile

\subsection{6/lupus-2019-Ism.126}

Background Studies looking for clinical association of antiribosomal P (anti-P) autoantibodies and lupus nephritis (LN) describe contradictory results. It is clear that anti-dsDNA antibodies contribute to $\mathrm{LN}$ pathogenesis and their titers fluctuate together with those of anti-P, suggesting a linked generation
Abstract 126 Table 1 Lupus nephritis histology class and antidsDNA and anti-P presence

\begin{tabular}{|c|c|c|c|c|}
\hline & & \multicolumn{3}{|c|}{ Autoantibodies } \\
\hline & & $\begin{array}{c}\text { Anti-dsDNA } \\
\text { present and no } \\
\text { anti-P }\end{array}$ & $\begin{array}{l}\text { Anti-dsDNA and } \\
\text { anti-P present }\end{array}$ & $\begin{array}{c}\text { Anti-dsDNA } \\
\text { and anti-P } \\
\text { absent }\end{array}$ \\
\hline $\begin{array}{l}\text { Lupus Nephritis Class } \\
\text { ISN/RPS }\end{array}$ & $n=26$ & $n=20$ & $n=4$ & $n=2$ \\
\hline Proliferative Class III or IV & 17 & 14 & 3 & 0 \\
\hline $\begin{array}{l}\text { Mixed Proliferative Class } \\
\text { III or IV and Membranous } \\
\text { Class V }\end{array}$ & 7 & 5 & 1 & 1 \\
\hline $\begin{array}{l}\text { Membranous Class V } \\
\text { "pure" }\end{array}$ & 2 & 1 & 0 & 1 \\
\hline
\end{tabular}

or an anti-dsDNA cross-reaction with the $\mathrm{P}$ antigen. We reexamined the anti-P involvement in $\mathrm{LN}$ in relation with the possibility of anti-dsDNA and anti-P cross-reactivity

Methods Anti-P and anti-dsDNA were analyzed by ELISA. SLE sera $(n=24)$ from patients with and with no $\mathrm{LN}$ were divided into 4 groups: A (anti-dsDNA positive, anti-P negative), B (both positive), C (anti-dsDNA negative, anti-P positive) and D (both negative). Anti-dsDNA cross-reaction was assessed against recombinant wild type and P-epitope-lacking P0 proteins using purified IgGs from SLE patients. Anti-P cross-reaction with dsDNA was analyzed testing affinity purified anti-P antibodies with an anti-dsDNA ELISA. LN biopsies $(n=26)$ were classified according to ISN/RPS and relations to the antidsDNA and anti-P simultaneous presence were examined

Results Neither anti-dsDNA nor anti-P antibodies showed evidence of cross-reaction in any of the applied tests. LN biopsy proven patients: age (media \pm SD) $34 \pm 11$ years; $88 \%$ female. No LN histologic class with anti-P relationship was observed: 9 patients had Class V (pure $\mathrm{n}=2$ ) or mixed with Class III/IV $(n=7) ; 1$ patient was anti-P positive ( $p$ value $>0.05)$. Two patients with LN had neither anti-dsDNA nor anti-P antibodies. The NIH Activity Index $(8.9 \pm 4.9)$ and NIH chronicity index scores $(1 \pm 1.1)$ and tubule-interstitial lesions were similar between anti-P positive or negative $\mathrm{LN}$ patients

Conclusions Cross-reactivity between anti-dsDNA and anti-P antibodies cannot explain the contradictory results of anti-P association with LN. Based on the present and our previous studies failing to find anti-P association with $\mathrm{LN}$, it seems unlikely that anti-P contribute to the renal damage. Other factors beyond anti-dsDNA and anti-P might participate in LN Funding Source(s): FONDECYT grant \# 1160513 and CONICYT Basal grant \# AFB170005

\section{HEPATIC INVOLVEMENT AS A PRESENTATION IN PEDIATRIC LUPUS: A RETROSPECTIVE STUDY OF 3 CASES}

${ }^{1}$ Ankita Singh* ${ }^{2}$ Gummadi Anjani, ${ }^{2}$ Rakesh Pilania, ${ }^{1}$ Ankur Jindal, ${ }^{2}$ Pandiarajan Vignesh, ${ }^{1}$ Deepti Suri, 'Surjit Singh. ${ }^{1}$ Postgraduate Institute of Medical Education and Research, Chandigarh, India; ${ }^{2}$ Dept. of Pediatrics, Allergy- Immunology Unit, Postgraduate Institute of Medical Education and Research

\subsection{6/lupus-2019-Ism.127}

Background Though abnormal liver tests can be seen during the course of disease in lupus, liver involvement as a 


\begin{tabular}{llll} 
Abstract 127 Table 1 & (Investigations) & & \\
\hline Investigation & Case 1 & Case 2 & Case 3 \\
\hline Haemoglobin (g/L) & 93 & 96 & 75 \\
White cell counts (IL) & $9.5 \times 10^{6}$ & $3.5 \times 10^{6}$ & $4.8 \times 10^{6}$ \\
Lymphocyte count (/L) & $1.9 \times 10^{6}$ & $1.2 \times 10^{6}$ & $0.3 \times 10^{6}$ \\
Platelet count (/L) & $145 \times 10^{9}$ & $147 \times 10^{9}$ & $37 \times 10^{9}$ \\
Total serum bilirubin (mg/dl) & 13.8 & & 7.1 \\
Conjugated bilirubin (mg/dl) & 12 & & 4 \\
Aspartate aminotransferase (IU/L) & 329 & 203 & 69 \\
Alanine aminotransferase (IU/L) & 96 & 156 & 57 \\
Urine protein (mg/m²/hour) & & $24 \mathrm{mg} / \mathrm{m}^{2} / \mathrm{hr}$ & \\
C3 (Normal 50-150 mg/dL) & 68 & 23 & 68 \\
C4 (Normal 20-50 mg/dL) & 4 & 4 & 3 \\
ANA & $3+$ diffuse & $3+$ diffuse & $4+$ speckled \\
Anti dsDNA (N:<60 IU/mL) & 733 & 1621 & 689 \\
\hline
\end{tabular}

presenting manifestation is uncommon in children with lupus.

Methods We retrospectively studied 140 pediatric lupus patients from January 1993- November 2018 and collected clinical and laboratory data of patients (3) who had liver involvement as presenting manifestation.

Results Case 1 was 13-year-old girl with fever and joint pain for 7 months associated with rash and yellow discoloration of eyes and body for 1 month. She also had altered behavior for 3 days. Examination revealed malar rash, pallor, jaundice, bilateral knee arthritis and hepatosplenomegaly. Investigations revealed (table 1) anemia, thrombocytopenia and lymphopenia. She had conjugated hyperbilirubinemia. Transaminases were elevated. Further investigations confirmed diagnosis of lupus (table 1). Markers for autoimmune hepatitis (SMA; LKM) were negative. She received pulse methylprednisolone followed by tapering doses of oral prednisolone. In view of neurological involvement she also received cyclophosphamide and shifted to azathioprine later. Her initial SLEDAI-2k was 22, that decreased to 4 at 2 year follow-up.

Case 2 was a 11-year-old boy with fever, rash and joint pain for 1 month. Examination revealed pallor, malar rash, oral ulcers, arthritis of bilateral elbow and knee and hepatomegaly. Investigations (table 1) revealed anemia; thrombocytopenia; leucopenia and lymphopenia. In view of multisystem involvement a possibility of lupus was considered which was confirmed by investigations (table 1). He had elevated transaminases. There was no coagulopathy. He was started on oral prednisolone. She also had class 3 lupus nephritis and received pulse cyclophosphamide followed by azathioprine in maintenance. Her transaminases decreased in follow-up. Her initial SLEDAI-2k was 18 , that decreased to 2 at 2 year follow-up.

Case 3 was an 9-year-old girl who had rash and abdominal distention for 20 days and fever and altered sensorium for 6 days. On examination she had pallor, malar rash, ascitis and hepatosplenomegaly. Investigations revealed anemia; thrombocytopenia; leucopenia and lymphopenia. She had conjugated hyperbilirubinemia, elevated transaminases and coagulopathy. She also had deranged renal function tests. Ultrasonography reveals coarse echotexture, gross ascitis and splenomegaly. Possibility of lupus with acute on chronic liver decompensation was considered which was confirmed by investigations (table 1). She was given methyl prednisolone. She had progressive azotemia so possibility of rapidly progressive renal failure was considered and cyclophosphamide was given. However, she developed refractory shock and eventually expired.

Conclusions Hepatic involvement at presentation in lupus can be multifaceted and poses challenge in diagnosis.

Funding Source(s): None

\section{THE LUPUS SEVERITY INDEX IS A PREDICTOR OF DAMAGE AND DEATH IN LUPUS PATIENTS}

${ }^{1}$ Paul R Fortin*, ${ }^{2}$ Anne-Sophie Julien, ${ }^{3}$ Ann E Clarke, ${ }^{4}$ Janet Pope, ${ }^{5}$ Mark Matsos, ${ }^{6}$ Douglas Smith, ${ }^{7}$ Christine Peschken. ${ }^{1} \mathrm{CHU}$ de Québec - Université Laval; ${ }^{2}$ Université Laval; ${ }^{3}$ Division of Rheumatology, Cumming School of Medicine, University of Calgary: ${ }^{4}$ University of Western Ontario; ${ }^{5}$ McMaster University; ${ }^{6}$ University of Ottawa; ${ }^{7}$ Faculty of Medicine, Department of Internal Medicine, University of Manitoba

\subsection{6/lupus-2019-Ism. 128}

Background Predictors of poor outcome in systemic lupus erythematosus (SLE) may lead to the identification of high-risk patients at the onset of disease (incident cases) and/or when we first assess them in our clinics (prevalent cases). We tested whether the Lupus Severity Index (LSI) can help characterize high versus low risk lupus patients.

Methods Population: Patients from six lupus centers were recruited according to a standard data collection protocol. We characterized incident cases and prevalent cases as those with a diagnosis made within or after the previous 15 months. Data collected: Demographic, socioeconomic, disease specific and medication data were collected at baseline and annually. We collected: the American College of Rheumatology (ACR) and the Systemic Lupus International Collaborating Clinics (SLICC) classification criteria, the SLE Disease Activity Index (SLEDAI), the Systemic Lupus Activity Questionnaire (SLAQ), and the SLICC Damage Index (SDI). The LSI was derived from the ACR classification criteria and used as a predictor variable. Statistical analyses: Kruskal-Wallis test and Spearman correlations were used to see the association of LSI with categorical and continuous variables respectively. The baseline LSI was used to predict outcomes at follow-ups using logistic regressions and Spearman correlations for dichotomous and continuous variables respectively.

Results We enrolled 639 lupus patients and 440, 324 and 168 were re-evaluated at 1,2 and 3 years. Baseline characteristics (table 1) [median (IQR)] were: age $=49.0 \quad(36.8-$ 58.5 ) years, female $=92 \%$, Caucasian $=74 \%$, disease duration=10.1 (2.7-20.6) years. We had 129 (20\%) incident cases and 471 (74\%) prevalent cases with missing information in 39 (6\%). Twelve patients died during follow-up. Table 1 summarizes baseline associations between LSI and several characteristics for the incident and prevalent cases. We found that age, sex, ethnicity (Asian worse LSI), SLICC classification criteria, SLEDAI, prednisone use and daily dose were associated with LSI in both incident and prevalent groups while the SDI and the use of immunosuppressors drugs was associated with LSI only in the prevalent cases. In follow-up, baseline LSI predicted SDI in prevalent cases $(p=0.02)$ with a trend in incident cases $(p=0.07)$. LSI predicted death in the prevalent group.

Conclusions The LSI is easy to derive from the ACR classification criteria and a useful measure of severity in lupus. The LSI is associated with baseline characteristics, some of them - 